\title{
Theaflavin digallate inactivates plasminogen activator inhibitor: Could tea help in Alzheimer's disease and obesity?
}

\author{
EWA SKRZYPCZAK-JANKUN ${ }^{1}$ and JERZY JANKUN ${ }^{1,2}$ \\ ${ }^{1}$ Urology Research Center, Department of Urology, University of Toledo - Health Science Campus, \\ 3000 Arlington Ave., Toledo, OH 43614, USA; ${ }^{2}$ Department of Clinical Nutrition, \\ Medical University of Gdansk, Debinki 7, 80-211 Gdansk, Poland
}

Received March 11,2010; Accepted April 14, 2010

DOI: 10.3892/ijmm_00000433

\begin{abstract}
Proteolysis in general and particularly the serine proteases are causally involved in many physiological processes and different diseases. Recently it was reported that plasminogen activator inhibitor type one (PAI-1) inactivation can alleviate the symptoms of Alzheimer's disease and reduce the body weight of obese individuals. In our broad search for natural compounds and their derivatives that can inhibit PAI-1, we include the polyphenols of teas since teas (green and black) or their components have been reported to alleviate the symptoms of both obesity and Alzheimer's disease. Inactivation of PAI-1 was measured in human plasma using thromboelastography. We used known PAI-1 inhibitor PAI039 [\{1-benzyl-5-[4-(trifluoromethoxy) phenyl]-1H-indol3-yl\}(oxo)acetic acid] as a positive control and (-)-epigallocatechin-3-gallate (EGCG), its prodrug octaacetate EGCG (OcAc EGCG) and theaflavin digallate [TH(2)] as potential PAI-1 inhibitors. We found that inactivation of PAI-1 in plasma by EGCG and OcAc EGCG was low or very low. However, TH(2) inactivated PAI-1 in a concentrationdependent manner with an $\mathrm{IC}_{50}$ of $18 \mu \mathrm{M}$ which is equal to or better than the $\mathrm{IC}_{50}$ reported for known PAI-1 inhibitor PAI039. Clearly TH(2) inhibits PAI-1 and might play a role in slowing down the progression of Alzheimer's disease or obesity by a PAI-1-dependent pathway. While the clinical value of $\mathrm{TH}(2)$ has not been proven, long-term prospective
\end{abstract}

Correspondence to: Professor Jerzy Jankun, Urology Research Center, Department of Urology, University of Toledo - Health Science Campus, 3000 Arlington Avenue, Toledo, OH 43614, USA E-mail: jerzy.jankun@utoledo.edu

Abbreviations: PAI-1, plasminogen activator inhibitor type 1; tPA, tissue plasminogen activator; uPA, urokinase plasminogen activator; PAI039, \{1-benzyl-5-[4-(trifluoromethoxy)phenyl]-1Hindol-3-yl\}(oxo)acetic acid; EGCG, (-)-epigallocatechin-3-gallate; Oc Ac EGCG, octaacetate EGCG; TH(2), theaflavin digallate; $\mathrm{AD}$, Alzheimer's disease; DMSO, dimethylsulfoxide

Key words: tea, polyphenols, plasminogen activator inhibitor type 1 studies assessing its efficacy are warranted due to the benign nature of the substance.

\section{Introduction}

Proteolysis in general and particularly the serine proteases are primary targets in drug discovery and development (1-3). These enzymes are causally involved in many physiological processes and different diseases including cancer, inflammation and blood disorders $(4,5)$. In certain diseases, proteases or their inhibitors are prognostic markers $(6,7)$ as in the case of the plasminogen activation system and breast cancer (4). Also, overexpression or low expression of tissue and urokinase plasminogen activators (tPA and uPA) or their inhibitors (mostly plasminogen activator inhibitor type 1; PAI-1) was reported to be critical in diseases. For example, a high level of urokinase is considered as a marker of unfavorable prognosis in breast cancer, while low levels of the plasminogen activator inhibitor (PAI-1) lead to extended bleeding which in some cases can be life-threating $(4,8)$. On the other hand low levels of tPA and high levels of PAI-1 are reported to be involved in deep vein thrombosis (9).

Recently it has been reported that PAI-1 and tPA are implicated in Alzheimer's disease. Evidence suggests that defects in amyloid peptide degradation are at the base of sporadic Alzheimer's disease (AD). Among the enzymes which can degrade amyloid peptide, plasmin - the serine protease activated by tPA - is particularly relevant. Low levels of plasmin activity have been detected in areas of AD human brains with a high susceptibility to amyloid plaque accumulation (10). Other researchers have demonstrated that plasmin cleaves $\beta$-amyloid at certain sites and that exogenously added plasmin blocks $\beta$-amyloid neurotoxicity, supporting a physiological role for plasmin in this disease $(11,12)$. Periz and Fortini suggested that $\beta$-amyloid levels are normally kept within physiological limits by regulated degradation involving a normal level of plasmin. However, high $B$-amyloid levels are able to overcome this system by inflammation-related tPA inhibition, consequently suppressing plasmin activity and promoting plaque formation (13). Cacquevel et al found that defective amyloid peptide degradation results, respectively, from a decrease in tPA expression and from an increase in the production of PAI-1 which further diminishes tPA-induced plasmin activity (14). 
Thus inhibition of PAI-1 activity can protect residual tPA which results in the degradation of amyloid plaque.

The other promising therapeutic application of PAI-1 inhibitors is obesity. It was reported that in visceral and subcutaneous adipose tissue PAI-1 mRNA expression is positively correlated with body mass index (BMI). Similar results have been found in plasma where PAI-1 activity/ antigen are positively and moderately associated with BMI. Furthermore, weight reduction substantially reduces plasma PAI-1 in obese humans $(15,16)$. It has also been shown that inhibitors of PAI-1 attenuate dietary fat-induced obesity in C57BL/6 mice. Specifically, it has been demonstrated in vivo that PAI-1 inhibition has a dose dependent effect on the reduction of body weight, adipocyte volume and circulating active PAI-1 in plasma $(15,17)$. There is no clear mechanism how inhibition of PAI-1 can reduce body weight. However, Crandall et al postulate three possibilities. First, inactivation of PAI-1 may stimulate migration of preadipocytes which would prevent their full differentiation into mature fat cells. Alternatively, PAI-1 inhibition may block angiogenesis that weakens vascularization and consequently the growth of adipose tissue. Finally, PAI-1 may affect fat tissue growth by altering receptordependent transport of lipids into lipocytes (17).

Thus, management of PAI-1 activity can be therapeutically significant in different diseases. In our broad search for natural compounds and their derivatives that can inhibit PAI-1 we include the polyphenols of tea since tea or its components can alleviate the symptoms of both obesity and Alzheimer's disease (18-21). However, the mechanisms by which tea and epigallocatechin-3-gallate (EGCG) lower body fat and are neuroprotective in Alzheimer's disease are not completely understood. We hypothesize that PAI-1 is inhibited by tea polyphenolic compounds which alleviate the symptoms of these diseases by a PAI-1-dependent pathway.

\section{Materials and methods}

Pooled normal human plasma was collected in sodium citrate and was rapidly processed, buffered and freeze-dried in a manner to assure the stability of hemeostasis proteins (product no. $258 \mathrm{~N}$; American Diagnostica, Stamford, CT). All homeostasis proteins were in the normal range. Lyophilized plasma was stored at $4^{\circ} \mathrm{C}$, and the aliquoted plasma was reconstituted in individual vials with $1.0 \mathrm{ml}$ of filtered deionized water. The vials were allowed to stand at room temperature with occasional swirling for 15-20 min before use.

Expression and purification of VLHL PAI-1. The mutation of two amino acids (Gln197 $\rightarrow$ Cys, Gly355 $\rightarrow$ Cys) in human wPAI-1 [SwissPROT P05121 (22)] produces VLHL PAI-1 with a half-life $>700 \mathrm{~h}$. The composition of this protein and the purification were previously described by our group $(23,24)$. VLHL PAI-1 was chosen to assure stable tPA inhibitory activity over time, which cannot be achieved when wild PAI-1 is used, since its activity changes significantly in a short time.

Tissue plasminogen activator. Fully active human tPA (product no. HTPA-TC) was purchased from Molecular Innovations, Novi, MI.
PAI-1 inhibitors. PAI039 [\{1-benzyl-5-[4-(trifluoromethoxy) phenyl]-1H-indol-3-yl\}(oxo)acetic acid] was purchased from Axon Medchem BV, Groningen, The Netherlands. Octaacetate (-)-epigallocatechin-3-gallate (OcAc EGCG) was a generous gift from PharmaIP LCC, Greenwich, CT, and (-)-epigallocatechin-3-gallate (EGCG) (catalog no. E4143) and theaflavins (catalog no. T5550) were purchased from Sigma-Aldrich, USA. E4143 is a mixture ( $\geq 80 \%$ of its content) of theaflavin, theaflavin gallate and theaflavin digallate.

SDS PAGE analysis. Electrophoresis of proteins was performed at room temperature using 4-12\% SDS-polyacrylamide gradient gels under non-reducing conditions. Gels were stained with Colloidal Coomassie Blue (Invitrogen, Grand Island, NY).

PAI-1/tPA complex formation assay. VLHL PAI-1 (24 $\mu 1$ of $0.4 \mathrm{mg} / \mathrm{ml}$ in PBS) and $5 \mu \mathrm{l}$ of DMSO or the appropriate inhibitor $(2.5 \mathrm{mg} / \mathrm{ml})$ in DMSO was added and incubated for $15 \mathrm{~min}$ at $37^{\circ} \mathrm{C}$. Immediately after incubation, $3 \mu 1$ of tPA $(2.1 \mathrm{mg} / \mathrm{ml}$ in 0.4 M HEPES and $0.1 \mathrm{M} \mathrm{NaCl}$; pH 7.4) was added and incubated for $15 \mathrm{~min}$ at $37^{\circ} \mathrm{C}$. Samples were run on a PAGE gel and stained. The optical band intensity of the formed tPA/VLHL PAI-1 complex was measured using densitometry and normalized to the VLHL PAI-1 band.

Analysis of plasma clot formation with thromboelastography. Thromboelastography, not only allows for the measurement of a global coagulation profile, but also yields data on the kinetics and dynamics of clot formation and clot lysis in whole blood or in plasma $(25,26)$. The critical part of this instrument is a pin hanging on a torsion wire and suspended in a cup holding a sample $(360 \mu 1)$. This pin oscillates at $6 \mathrm{rpm}$ at a $4^{\circ} 45^{\prime}$ angle at $37^{\circ} \mathrm{C}$. When plasma changes viscosity during clot formation this pin motion is progressively restrained by the clot and the cup. The strength of the clot determines the degree of force on the pin. The pin movement is converted to a TEG trace (27). Sodium citrated, reconstituted plasma was used for TEG assays by mixing $1 \mathrm{ml}$ of plasma with $20 \mu \mathrm{l}$ of kaolin (Haemoscope Co., Neils, IL) to which a constant amount of tPA was added $[10 \mu 1$ of tPA $(2.1 \mathrm{mg} / \mathrm{ml}$ in 0.4 M HEPES, 0.1 M NaCl; $\mathrm{pH}$ 7.4)] as a fibrinolytic agent $(28,29)$ to measure proteolysis under controlled conditions $(23,26,30)$. Next, $320 \mu 1$ of the mixture was transferred to each TEG cup containing $20 \mu \mathrm{l}$ of $\mathrm{CaCl}_{2}(0.2 \mathrm{M})$ and an activity assay buffer (50 mM HEPES, $150 \mathrm{mM} \mathrm{NaCl}, 1 \%$ human serum albumin, $0.05 \%$ Tween-20 buffer, $\mathrm{pH}$ 6.6) with i) VLHL PAI-1 to prevent lysis by tPA, or ii) VLHL PAI-1 plus tested compound to check its inhibitory action demonstrated by lysis of the clot when tPA is unopposed by PAI-1 activity. The critical parameters of clotting measured by TEG are as follows: $\mathrm{R}$ is the time from the start of the reaction until a measurable clot is detected, $\mathrm{K}$ is the time from the $\mathrm{R}$ point until a certain clot firmness is achieved, $\alpha$ is the maximum angle that represents kinetics of clotting and LY30 (percentage) represents clot lysis $30 \mathrm{~min}$ after MA (maximum amplitude). A typical thromboelastogram is shown in Fig. 1. 


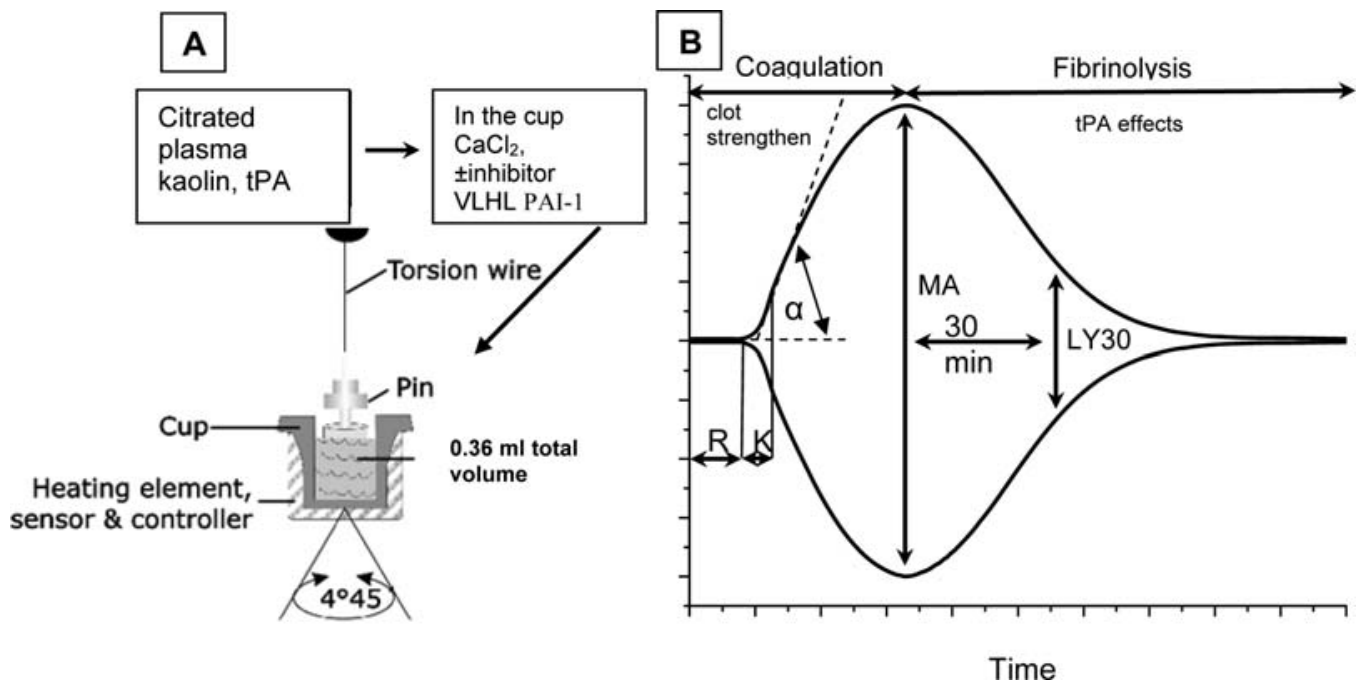

Figure 1. (A) Design of the thromboelastograph cup and pin. (B) Graphical representation of the plasma clotting process. Specific determinants are as follows: reaction time $\mathrm{R}$ is the time from the start of the reaction until a measurable clot is detected; $\mathrm{K}$ is the time from the $\mathrm{R}$ point until a certain clot firmness is achieved; the angle $\alpha$ or An reflects the rate of clot formation; the maximum amplitude MA of clot shear elasticity reflects the contribution of fibrin to clot strength, and LY30 is the amplitude at $30 \mathrm{~min}$ after MA, which reflects the speed of fibrinolysis.

\section{Results}

VLHL PAI-1 and tPA form a complex observed on SDS PAGE gel as a single band of molecular weight equal to the sum of the MW for both proteins. Addition of inhibitors reduces the intensity of this band. We measured it by densitometry and normalized it to the intensity of the VLHL PAI-1 band. As noted in Fig. 2, PAI039 inhibited the complex formation almost completely, while the other agents were less efficient but progressively more effective in the following order: OcAc EGCG $<$ EGCG $<$ TH(2). However, Gorlatova et al reported that PAI039 exhibited reversible inactivation of PAI-1, and the site of interaction of the PAI039 drug with the PAI-1 molecule is inaccessible when PAI-1 is bound to vitronectin and may overlap with the PAI-1 vitronectin binding domain (31). Human vitronectin is a major plasma glycoprotein and thus could interfere with the inhibition of PAI-1 by inhibitors identified in vitro.

Consequently, all experiments involving PAI-1 inhibition were carried out with normalized human plasma, and kinetics of clot formation and its lysis were measured by thromboelastography. Normalized human plasma clots after addition of $\mathrm{Ca}^{++}$and kaolin, tPA activates plasminogen to lyse the clot, while VLHL PAI-1 inhibits tPA thus protecting the clot

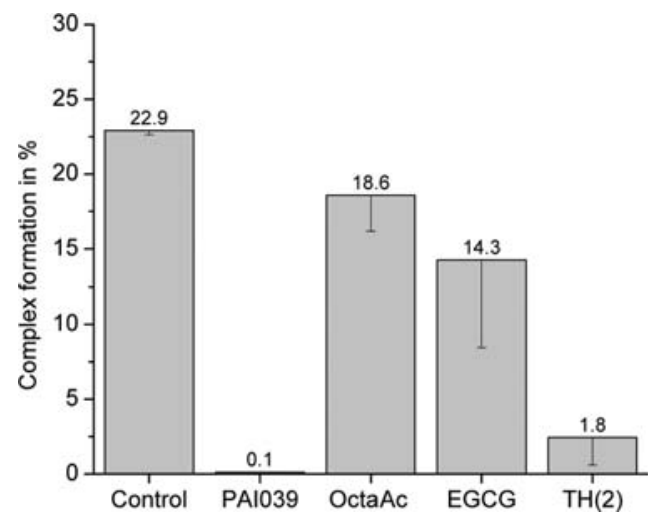

Figure 2. Inhibition of tPA/VLHL PAI-1 complex formation as measured by its band intensity on SDS-PAGE gel for different PAI-1 inhibitors.

from tPA-induced lysis. Inhibitors of PAI-1 reduce VLHL PAI-1 protection and allow complete or partial tPA-induced lysis. Expected thromboelastograms are documented in Table I.

To standardize our data, all potential inhibitors were dissolved in DMSO. In our previous study we found that DMSO altered various properties of PAI-1 (for example, its

Table I. Changes in the thromboelastogram as a function of proteolysis status.

\begin{tabular}{|c|c|c|c|c|}
\hline & Control & tPA treated & tPA + VLHL PAI-1 & $\begin{array}{l}\text { tPA + VLHL PAI-1 } \\
+ \text { Inhibitor }\end{array}$ \\
\hline tPA & - & + & + & + \\
\hline VLHL PAI-1 & - & - & + & + \\
\hline Inhibitor & - & - & - & + \\
\hline $\begin{array}{l}\text { Expected } \\
\text { thrombo- } \\
\text { elastogram }\end{array}$ & & & & \\
\hline
\end{tabular}



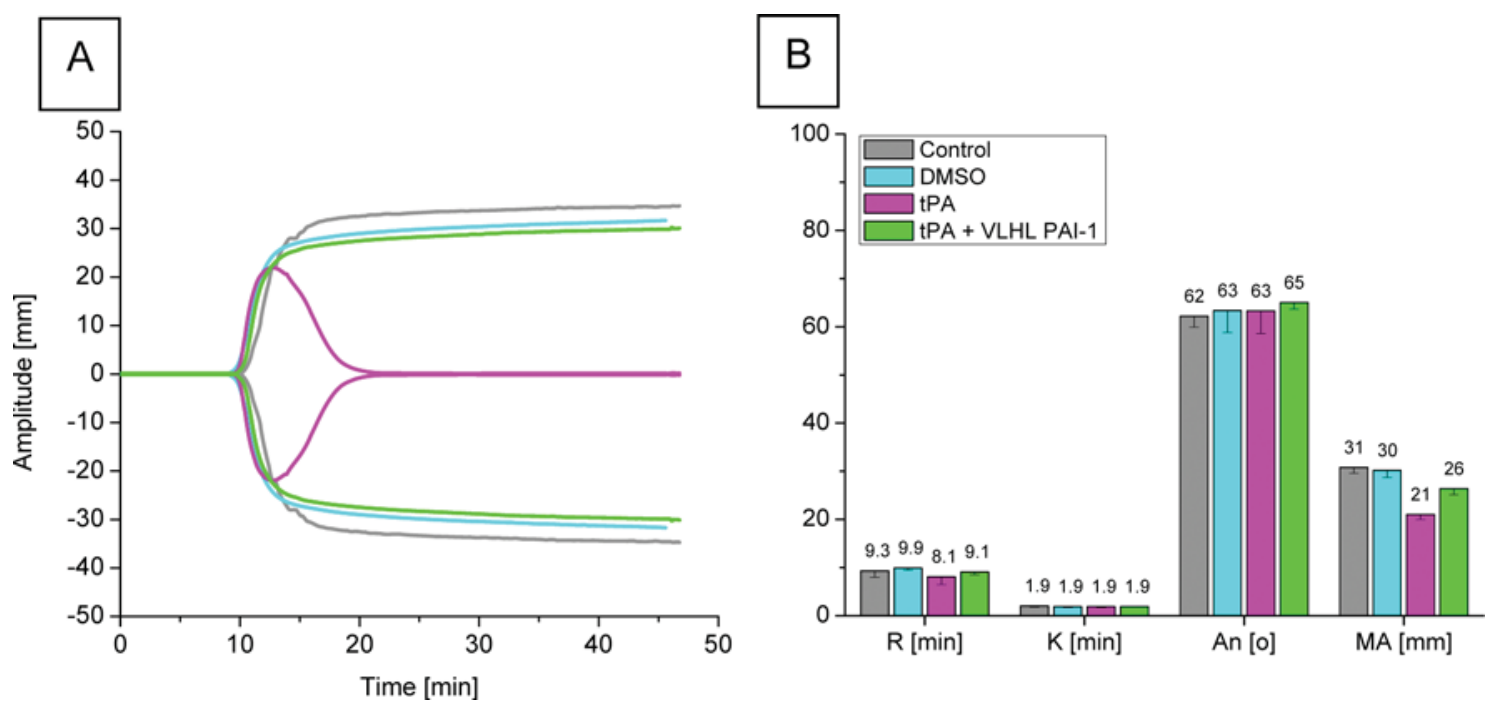

Figure 3. (A) Example of a thromboelastogram of clotted plasma (grey, control) with DMSO (light blue); treated with DMSO and tPA (magenta); treated with DMSO, tPA and VLHL PAI-1 (green). (B) Selected parameters of plasma clotting; the median of four independent experiments. Color scheme identical as in A. $n=4$.
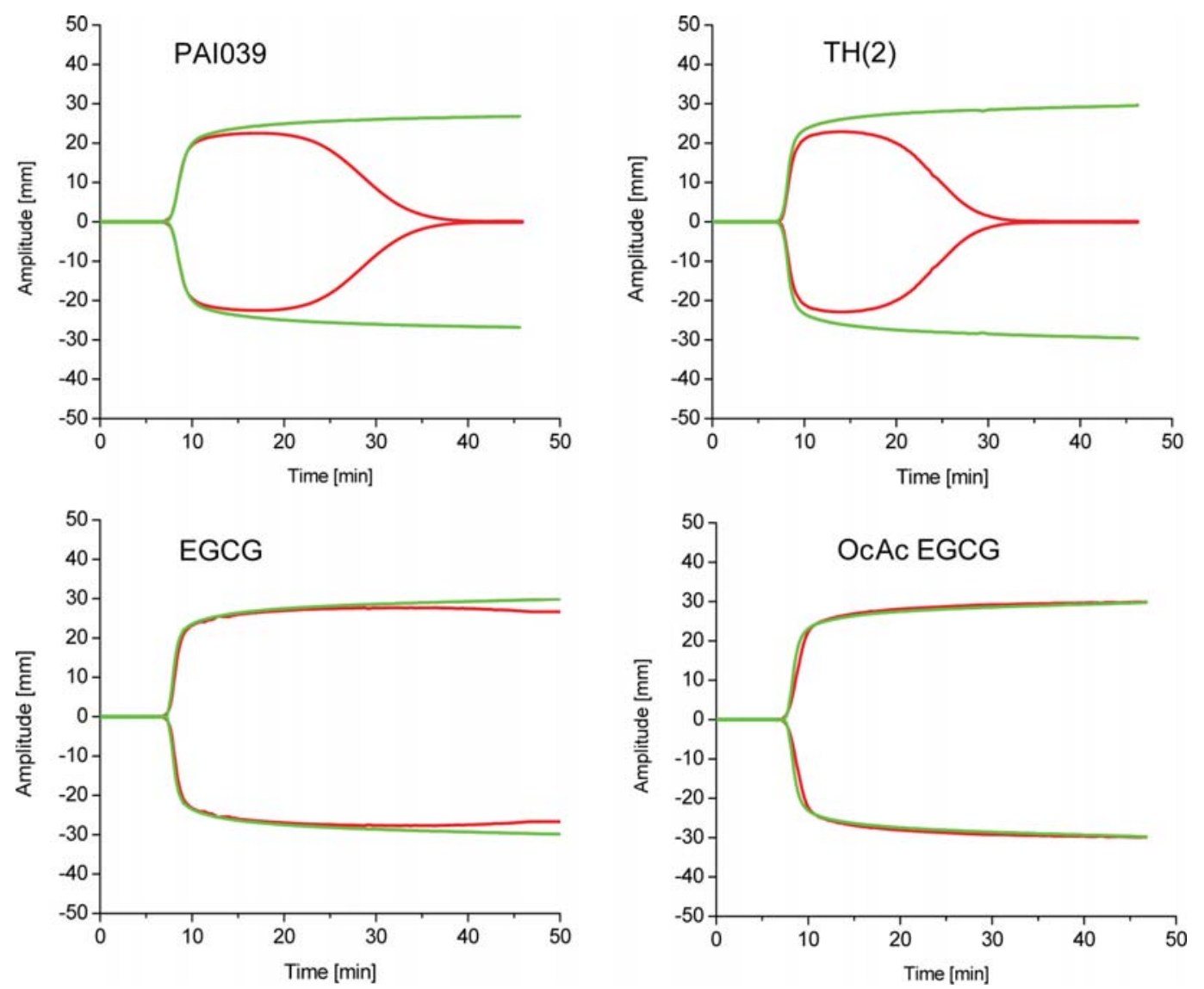

Figure 4. Examples of thromboelastograms of different PAI-1 inhibitors. The concentrations of the inhibitors were: PAI039, $15 \mu \mathrm{M}$; TH(2), $11 \mu \mathrm{M}$; EGCG, $17 \mu \mathrm{M}$; OcAc EGCG, $15 \mu \mathrm{M}$.

electrophoretic mobility) (32). Hence we investigated whether addition of DMSO alters the clotting characteristics of plasma. Differences in all of the determined parameters between DMSO-treated and control samples were not statistically significant (Fig. 3). Thus, we concluded that
DMSO does not change the clotting parameters of plasma. In the same experiment we assessed how tPA would perform alone and when opposed by VLHL PAI-1 in the presence of DMSO. Most clotting parameters for tPA or tPA+VLHL PAI-1 were within experimental errors. However, we observed 


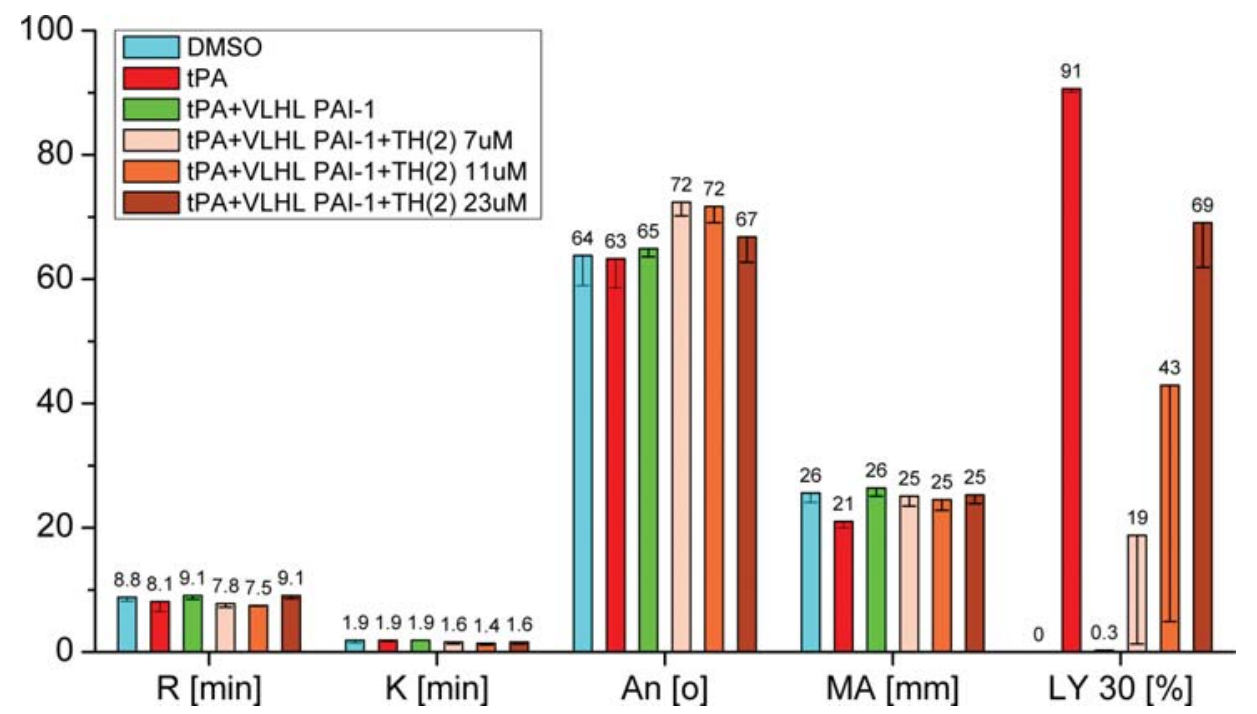

Figure 5. Clotting parameters of plasma treated with tPA; tPA+VLHL PAI-1; and tPA+VLHL PAI-1 with theaflavin digallate [TH(2)] in different concentrations $(n=4)$.

statistically significant lower MA (represents clot strength) for tPA-treated samples $(\mathrm{P}<0.05)$ (Fig. 3). Furthermore, LY30 for tPA was $90.7 \pm 0.7 \%$ while addition of VLHL PAI-1 decreased LY30 to $0.3 \pm 0.9 \%$. LY30 for all other samples (control, DMSO) was 0 indicating lack of inhibitory activity toward PAI-1.

PAI039 inhibited PAI-1 in human plasma as determined by thromboelastography. Even more potent was theaflavin digallate while EGCG showed much less PAI-1 inhibitory activity. No activity was observed for OcAc EGCG under the same conditions.

Inhibition of PAI-1 by $\mathrm{TH}(2)$ was concentration-dependent as illustrated in Fig. 4. The calculated $\mathrm{IC}_{50}$ in plasma was $18 \mu \mathrm{M}$. All other clotting parameters (Fig. 5) were within experimental errors with the exception of An $(\alpha)$ which was slightly elevated for two lower concentrations of $\mathrm{TH}(2)$. The $\alpha$ angle is an indication of the rate of clot formation (33), but explanation of the possible mechanism is highly speculative and was outside the scope of this study.

\section{Discussion}

Green tea polyphenol EGCG and black tea polyphenols are traditionally perceived as simple radical scavengers, although a growing body of evidence reveals their previously unsuspected biochemical activity $(4,34,35)$. The PAI- 1 inhibitory activity of theaflavin digallate falls into this category. Inhibition of PAI-1 by tea polyphenols has not been reported in the scientific literature to date. Yet, inhibition of PAI-1 by EGCG among the other compounds was quoted in a 2009 patent application (36) independently confirming our findings.

Of the 4 tested compounds $\mathrm{TH}(2)$ showed the highest PAI-1 inactivation. PAI039 with a $\mathrm{IC}_{50}$ reported by different authors between 2.7 (37) and 10-20 $\mu \mathrm{M}$ (36) was used as a positive control. $\mathrm{IC}_{50}$ in plasma for $\mathrm{TH}(2)$ in our experiments was $18 \mu \mathrm{M}$. Inactivation of PAI-1 by PAI039, measured by thromboelastometry, was slightly less effective than $\mathrm{TH}(2)$ but clearly within the reported range $(36,37)$. The PAI-1 inhibitory activity of EGCG was much less effective that the two previously mentioned compounds. EGCG interacts with many targets including MAP kinases, phosphatases, DNA, RNA, DNA methyltransferase and topoisomerases (38). It has also been reported that the majority of such interactions result in inhibitory effects (38). Thus it is possible that the high PAI-1 inhibitory activity reported in isolated systems will somehow be lower when measured in plasma, where the abundance of different proteins can compete for EGCG binding. OcAc EGCG did not inhibit PAI-1 under the same conditions as TH(2). OcAc EGCG is considered a prodrug of EGCG to which OcAc EGCG hydrolyzes $(39,40)$. However, it must be stated that when the sensitivity of the assay was greatly increased, we observed some PAI-1 inhibitory activity for both compounds (data not shown).

In conclusion, $\mathrm{TH}(2)$ markedly inhibits PAI-1 and might play a role in slowing down the progression of Alzheimer's disease or obesity by a PAI-1-dependent pathway. While the clinical value of $\mathrm{TH}(2)$ has not been confirmed, long-term prospective studies evaluating its efficacy are warranted due to the benign nature of the substance. $\mathrm{TH}(2)$ can also be a leading compound for future development of more potent compounds.

\section{Acknowledgements}

We thank Dr R. Hart (President, PharmaIP LLC, Greenwich, CT, USA) for the helpful remarks and discussions, the support and the chemicals used in this study. This study was supported, in part, by grants from PharmaIP LLC and the Frank D. Stranahan Endowment Fund for Oncological Research.

\section{References}

1. Cudic M and Fields GB: Extracellular proteases as targets for drug development. Curr Protein Pept Sci 10: 297-307, 2009.

2. Eddy AA: Serine proteases, inhibitors and receptors in renal fibrosis. Thromb Haemost 101: 656-664, 2009. 
3. Struniawski R, Szpechcinski A and Chorostowska-Wynimko J: Molecular diagnostics of alpha-1-antitrypsin deficiency in clinical practice. Pneumonol Alergol Pol 76: 253-264, 2008.

4. Jankun J and Skrzypczak-Jankun E: Yin and yang of the plasminogen activator inhibitor. Pol Arch Med Wewn 119: 410-417, 2009

5. Martorell L, Martinez-Gonzalez J, Rodriguez C, Gentile M, Calvayrac $\mathrm{O}$ and Badimon L: Thrombin and protease-activated receptors (PARs) in atherothrombosis. Thromb Haemost 99: 305-315, 2008

6. Hildenbrand R, Schaaf A, Dorn-Beineke A, et al: Tumor stroma is the predominant uPA-, uPAR-, PAI-1-expressing tissue in human breast cancer: prognostic impact. Histol Histopathol 24: 869-877, 2009

7. Smith R, Xue A, Gill A, et al: High expression of plasminogen activator inhibitor-2 (PAI-2) is a predictor of improved surviva in patients with pancreatic adenocarcinoma. World J Surg 31 493-503, 2007.

8. Jankun J, Aleem AM, Struniawski R, Lysiak-Szydlowska W, Selman SH and Skrzypczak-Jankun E: Accelerated thrombus lysis in the blood of plasminogen activator inhibitor deficient mice is inhibited by PAI-1 with a very long half-life. Pharmacol Rep 61: 673-680, 2009.

9. Akhtar N, Deleu D and Kamran S: Haematologic disorders and cerebral venous thrombosis. J Pak Med Assoc 56: 498-501, 2006

10. Dotti CG, Galvan C and Ledesma MD: Plasmin deficiency in Alzheimer's disease brains: causal or casual? Neurodegener Dis 1: 205-212, 2004.

11. Tucker HM, Kihiko M, Caldwell JN, et al: The plasmin system is induced by and degrades amyloid-beta aggregates. J Neurosci 20: 3937-3946, 2000.

12. Van Nostrand WE and Porter M: Plasmin cleavage of the amyloid beta-protein: alteration of secondary structure and stimulation of tissue plasminogen activator activity. Biochemistry 38: 11570-11576, 1999.

13. Periz G and Fortini ME: Proteolysis in Alzheimer's disease. Can plasmin tip the balance? EMBO Rep 1: 477-478, 2000.

14. Cacquevel M, Launay S, Castel H, et al: Ageing and amyloidbeta peptide deposition contribute to an impaired brain tissue plasminogen activator activity by different mechanisms. Neurobiol Dis 27: 164-173, 2007.

15. Correia ML and Haynes WG: A role for plasminogen activator inhibitor-1 in obesity: from pie to PAI? Arterioscler Thromb Vasc Biol 26: 2183-2185, 2006.

16. Mavri A, Alessi MC, Bastelica D, et al: Subcutaneous abdominal, but not femoral fat expression of plasminogen activator inhibitor-1 (PAI-1) is related to plasma PAI-1 levels and insulin resistance and decreases after weight loss. Diabetologia 44: 2025-2031, 2001.

17. Crandall DL, Quinet EM, El Ayachi S, et al: Modulation of adipose tissue development by pharmacological inhibition of PAI-1. Arterioscler Thromb Vasc Biol 26: 2209-2215, 2006.

18. Bose M, Lambert JD, Ju J, Reuhl KR, Shapses SA and Yang CS: The major green tea polyphenol, (-)-epigallocatechin3-gallate, inhibits obesity, metabolic syndrome, and fatty liver disease in high-fat-fed mice. J Nutr 138: 1677-1683, 2008.

19. Chen N, Bezzina R, Hinch E, et al: Green tea, black tea, and epigallocatechin modify body composition, improve glucose tolerance, and differentially alter metabolic gene expression in rats fed a high-fat diet. Nutr Res 29: 784-793,2009.

20. Mandel SA, Amit T, Weinreb O, Reznichenko L and Youdim MB: Simultaneous manipulation of multiple brain targets by green tea catechins: a potential neuroprotective strategy for Alzheimer and Parkinson diseases. CNS Neurosci Ther 14: 352-365, 2008 .

21. Rezai-Zadeh K, Arendash GW, Hou H, et al: Green tea epigallocatechin-3-gallate (EGCG) reduces beta-amyloid mediated cognitive impairment and modulates tau pathology in Alzheimer transgenic mice. Brain Res 1214: 177-187, 2008.
22. Pannekoek H, Veerman H, Lambers H, et al: Endothelial plasminogen activator inhibitor (PAI): a new member of the Serpin gene family. EMBO J 5: 2539-2544, 1986

23. Jankun J, Aleem AM, Selman SH, et al: Highly stable plasminogen activator inhibitor type one (VLHL PAI-1) protects fibrin clots from tissue plasminogen activator-mediated fibrinolysis. Int J Mol Med 20: 683-687, 2007.

24. Jankun J, Aleem AM, Specht Z, et al: PAI-1 induces cell detachment, downregulates nucleophosmin (B23) and fortilin (TCTP) in LnCAP prostate cancer cells. Int J Mol Med 20: 11-20, 2007.

25. Evans PA, Hawkins K, Lawrence M, Barrow MS, Williams PR and Williams RL: Studies of whole blood coagulation by oscillatory shear, thromboelastography and free oscillation rheometry. Clin Hemorheol Microcirc 38: 267-277, 2008.

26. Gallimore MJ, Harris SL, Tappenden KA, Winter M and Jones DW: Urokinase induced fibrinolysis in thromboelastography: a model for studying fibrinolysis and coagulation in whole blood. J Thromb Haemost 3: 2506-2513, 2005.

27. Carroll RC, Craft RM, Chavez JJ, Snider CC, Kirby RK and Cohen E: Measurement of functional fibrinogen levels using the thrombelastograph. J Clin Anesth 20: 186-190, 2008.

28. Carr ME Jr, Krishnamurti C and Alving BM: Effect of plasminogen activator inhibitor-1 on tissue-type plasminogen activator-induced fibrinolysis. Thromb Haemost 67: 106-110, 1992.

29. Sugiki M, Maruyama M, Yoshida E, Mihara H, Kamiguti AS and Theakston DG: Enhancement of plasma fibrinolysis in vitro by jararhagin, the main haemorrhagic metalloproteinase in Bothrops jararaca venom. Toxicon 33: 1605-1617, 1995.

30. Kohro S, Yamakage M, Omote T and Namiki A: In vitro effects of propofol on blood coagulability and fibrinolysis by the use of thromboelastograph technique. Acta Anaesthesiol Scand 43: 217-219, 1999

31. Gorlatova NV, Cale JM, Elokdah H, et al: Mechanism of inactivation of plasminogen activator inhibitor-1 by a small molecule inhibitor. J Biol Chem 282: 9288-9296, 2007.

32. Jankun J, Aleem AM, Selman SH, Basrur V and SkrzypczakJankun E: VLHL plasminogen activator inhibitor spontaneously reactivates from the latent to active form. Int $\mathrm{J}$ Mol Med 23: 57-63, 2009.

33. Alexander DC, Butt WW, Best JD, Donath SM, Monagle PT and Shekerdemian LS: Correlation of thromboelastography with standard tests of anticoagulation in paediatric patients receiving extracorporeal life support. Thromb Res (In press).

34. Frank B and Gupta S: A review of antioxidants and Alzheimer's disease. Ann Clin Psychiatry 17: 269-286, 2005.

35. Kostrzewa RM and Segura-Aguilar J: Novel mechanisms and approaches in the study of neurodegeneration and neuroprotection. A review. Neurotox Res 5: 375-383, 2003.

36. Lawrence DA, Strickland D, Cale J, Su EJ, Emal C and Warnock M: Plasminogen activator inhibitor-1 inhibitors and methods of use thereof to modulate lipid metabolism. International Application No.: PCT/US2008/060542, The Regents of The University of Michigan, USA, 2009.

37. Elokdah H, Abou-Gharbia M, Hennan JK, et al: Tiplaxtinin, a novel, orally efficacious inhibitor of plasminogen activator inhibitor-1: design, synthesis, and preclinical characterization. J Med Chem 47: 3491-3494, 2004.

38. Patra SK, Rizzi F, Silva A, Rugina DO and Bettuzzi S: Molecular targets of (-)-epigallocatechin-3-gallate (EGCG): specificity and interaction with membrane lipid rafts. J Physiol Pharmacol 59 (Suppl 9): 217-235, 2008.

39. Chao J, Lau WK, Huie MJ, et al: A pro-drug of the green tea polyphenol (-)-epigallocatechin-3-gallate (EGCG) prevents differentiated SH-SY5Y cells from toxicity induced by 6hydroxydopamine. Neurosci Lett 469: 360-364, 2009.

40. Kok SH, Wong RS, Gambari R, et al: In vitro cytotoxicity of (-)-EGCG octaacetate on MDAMB-231 and SKHep-1 human carcinoma cells: A pharmacological consideration on prodrug design. Int J Mol Med 22: 841-845, 2008. 